\title{
Ultra-Thin Ultra-Smooth and Low-Loss Silver and Silver- Silica Composite Films for Superlensing Applications
}

\author{
Weiqiang Chen, Mark D. Thoreson, Alexander V. Kildishev and Vladimir M. Shalaev \\ Birck Nanotechnology Center and School of Electrical and Computer Engineering, Purdue University, West Lafayette, IN 47907, USA \\ wqchen@purdue.edu
}

\begin{abstract}
We demonstrate a method to fabricate ultra-thin ultra-smooth and low-loss silver and silver-silica composite films using a germanium wetting layer and a rapid post-annealing treatment. Such achievement satisfies both the demands for superlenses and hyperlenses.
\end{abstract}

(C)2010 Optical Society of America

OCIS codes: (160.3900) Metals; (310.3840) Materials and process characterization.

Recent advances in metamaterials have facilitated several new sub-diffraction resolution devices. Examples include the near-field superlens [1-3] and the far-field hyperlens [4-8] which have attracted a great deal of both theoretical and experimental research interest. Realization of superlens and hyperlens devices requires extremely smooth, thin and low loss metal films which form their basic structures [2-4]. Silver is the most often used metal material in recent superlens and hyperlens designs due to the fact that it exhibits the lowest on-resonance loss of any natural noble metal at optical frequencies [9]. However, silver and its dielectric composite films often exhibit high surface roughness in fabrication [3]. It is also difficult to achieve a uniformly continuous silver film at a thickness less than 20nm. These factors limit the ultimate resolution achievable with the superlens and the overall performance of hyperlens-type devices. Recent work has shown promise in using a germanium (Ge) wetting layer during the fabrication process to reduce the silver surface roughness [10]. In this work, we extended these studies and investigate the methods of fabricating ultra-thin, ultra-smooth and low-loss silver films and silver-silica composite films to address the demands for fine sub-wavelength resolution of superlens and hyperlens devices.

First, it was proved that an ultra-thin and ultra-smooth continuous silver film can be obtained using a Ge wetting layer in fabrication. The inclusion of the thin Ge layer as a wetting material acts to drastically reduce the percolation threshold of $\mathrm{Ag}$ films. The percolation threshold shifts from a silver layer thickness of about $11 \mathrm{~nm}$ (for Ag deposited on $\mathrm{SiO}_{2}$ via by e-beam evaporation [11]) to a thickness of about $3 \mathrm{~nm}$ when a Ge sublayer is included in the fabrication. Hence with a Ge wetting layer it is possible to make ultra-thin uniformly continuous Ag films at thicknesses of only $5.5 \mathrm{~nm}$. Through AFM analysis, it was confirmed that the addition of a Ge wetting layer reduces the surface roughness of the Ag films to around 0.2-0.7 $\mathrm{nm}$.

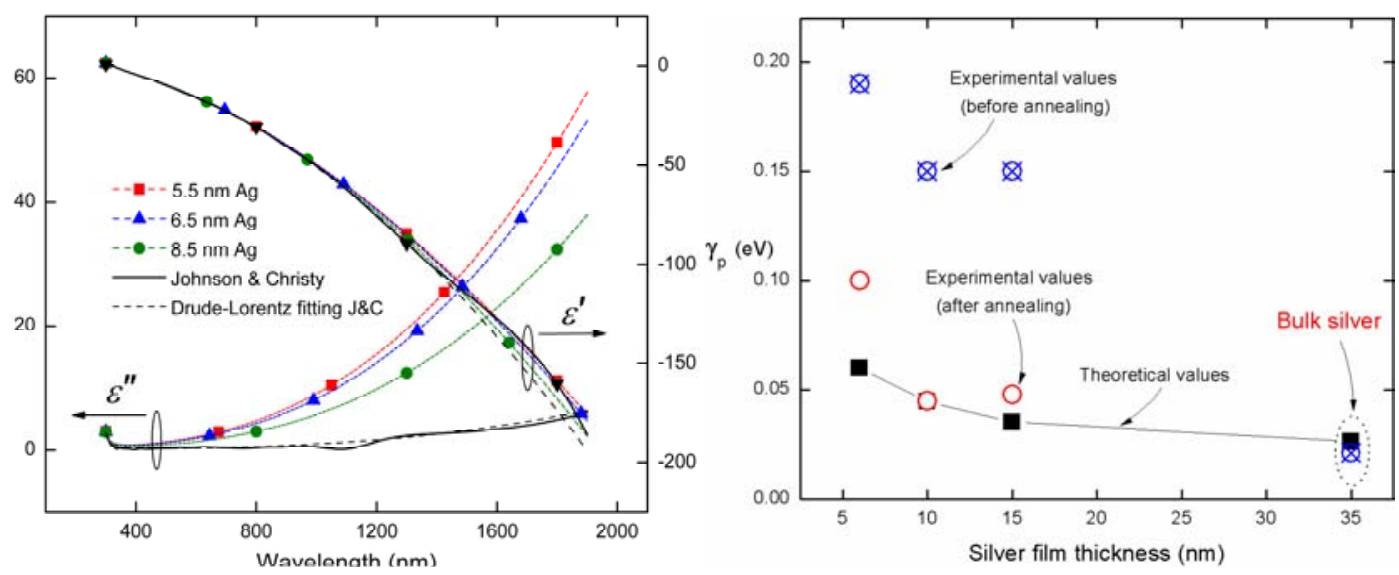

Fig. 1. (a) Retrieved complex permittivity curves from samples with 1-nm Ge wetting layers and those of bulk silver and a fitted Drude-Lorentz model $[14,15]$. (b) Theoretical calculated [13] and experimental retrieved damping constant $\left(\gamma_{\mathrm{p}}\right)$ curves of Ag films at different thickness on a 1-nm Ge wetting layer. 


\section{QThE4.pdf}

However, the ultra-thin ultra-smooth Ag films exhibit higher loss than bulk silver due to the quantum size effect $[12,13]$ as shown in Fig. 1(a). When the grain sizes in the film and the film thickness become extreme small, an additional size-dependent term in the loss should be included $[12,13]$. For the purpose of reducing the losses of the ultrathin Ag films, a rapid post-annealing treatment was introduced in our experiments to let the grains in polycrystalline or amorphous silver films to grow in size, thereby reducing the size-dependent term. This beneficial result of annealing must be carefully controlled and optimized to ensure that the silver film does not change into a semicontinous, island-like film, which can occur for annealing temperatures that are too high $\left(>350^{\circ} \mathrm{C}\right)$ or annealing time( $>6$ minutes) that are too long. Our experimental results in Fig. 1(b) show that the rapid post-annealing treatment is effective for $\mathrm{Ag}$ films with different thicknesses. For example, under optimized annealing conditions( 3 minutes, $280^{\circ} \mathrm{C}$ ), the loss of a $10-\mathrm{nm}$ thick $\mathrm{Ag}$ film can be reduced by $70 \%$ and at the same time maintain a low surface roughness in the sample. This annealing method is capable of reducing the losses of the silver films to the ideally possible values allowed by the quantum size effect in smaller grains, and no further improvement could be expected.

Overall, our results indicate that, ultra-thin, ultra-smooth and low-loss silver films can be achieved by introducing a very thin Ge wetting layer and a rapid post-annealing treatment. Ultra-smooth $\left(\mathrm{Ag} / \mathrm{Ge} / \mathrm{SiO}_{2}\right)_{\mathrm{n}}$ lamellar composite films with ultra-thin low-loss Ag sublayers were also achieved via the same wetting and annealing methods. This achievement satisfies both the superlens and hyperlens demands for high resolution sub-wavelength imaging.

\section{References}

[1] J. B. Pendry, "Negative refraction makes a perfect lens," Phys Rev Lett 85, 3966-3969 (2000).

[2] S. A. Ramakrishna, J. B. Pendry, M. C. K. Wiltshire, and W. J. Stewart, "Imaging the near field," Journal of Modern Optics 50, 1419-1430 (2003).

[3] N. Fang, H. Lee, C. Sun, and X. Zhang, "Sub-diffraction-limited optical imaging with a silver superlens," Science 308, 534-537 (2005).

[4] Z. W. Liu, H. Lee, Y. Xiong, C. Sun, and X. Zhang, "Far-field optical hyperlens magnifying sub-diffraction-limited objects," Science 315, 1686-1686 (2007).

[5] Z. Jacob, L. V. Alekseyev, and E. Narimanov, "Semiclassical theory of the hyperlens," Journal of the Optical Society of America a-Optics Image Science and Vision 24, A54-A61 (2007).

[6] A. V. Kildishev and V. M. Shalaev, "Engineering space for light via transformation optics," Optics Letters 33, $43-45$ (2008).

[7] A. V. Kildishev, U. K. Chettiar, Z. Jacob, V. M. Shalaev, and E. E. Narimanov, "Materializing a binary hyperlens design," Applied Physics Letters 94, 071102 (2009).

[8] A. V. Kildishev and E. E. Narimanov, "Impedance-matched hyperlens," Optics Letters 32, 3432-3434 (2007).

[9] P. B. Johnson and R. W. Christy, "Optical-Constants of Noble-Metals," Physical Review B 6, 4370-4379 (1972).

[10] L. Vj, N. P. Kobayashi, M. S. Islam, W. Wu, P. Chaturvedi, N. X. Fang, S. Y. Wang, and R. S. Williams, "Ultrasmooth Silver Thin Films Deposited with a Germanium Nucleation Layer," Nano Letters 9, 178-182 (2009).

[11] P. Nyga, V. P. Drachev, M. D. Thoreson, and V. M. Shalaev, "Mid-IR plasmonics and photomodification with Ag films," Appl Phys BLasers O 93, 59-68 (2008).

[12] V. P. Drachev, U. K. Chettiar, A. V. Kildishev, H. K. Yuan, W. S. Cai, and V. M. Shalaev, "The Ag dielectric function in plasmonic metamaterials," Optics Express 16, 1186-1195 (2008).

[13] A. Pinchuk, U. Kreibig, and A. Hilger, "Optical properties of metallic nanoparticles: influence of interface effects and interband transitions," Surface Science 557, 269-280 (2004).

[14] X. Ni, Z. Liu, and A. V. Kildishev, "PhotonicsDB: Optical Constants" (2007), DOI:10254/nanohub-r3692.5.

[15] S. Ishii, U. K. Chettiar, X. Ni, and A. V. Kildishev, "PhotonicsRT: Wave Propagation in Multilayer Structures" (2008), DOI:10254/nanohub-r5968.8. 